\title{
Quantifying real-time sample temperature under the gas environment in the transmission electron microscope using a novel MEMS heater
}

\author{
Meng Li ${ }^{1,3 *}$, De-Gang Xie ${ }^{1}$, Xi-Xiang Zhang ${ }^{2}$, Judith C. Yang ${ }^{3}$, Zhi-Wei Shan ${ }^{1 *}$ \\ ${ }^{1}$ Center for Advancing Materials Performance from the Nanoscale (CAMP-Nano), State Key \\ Laboratory for Mechanical Behavior of Materials, Xi'an Jiaotong University, Xi'an 710049, \\ China \\ ${ }^{2}$ Division of Physical Science and Engineering, King Abdullah University of Science \& \\ Technology (KAUST), Thuwal 23955-6900, Saudi Arabia \\ ${ }^{3}$ Department of Chemical and Petroleum Engineering, University of Pittsburgh, Pittsburgh \\ 15260, USA \\ *e-mail: zwshan@mail.xjtu.edu.cn (Z.S), mona.mengli@gmail.com (M.L.)
}




\begin{abstract}
Accurate control and measurement of real-time sample temperature are critical for the understanding and interpretation of the experimental results from in situ heating experiments inside environmental TEM (ETEM). However, quantifying the real-time sample temperature remains a challenging task for commercial in situ TEM heating devices, especially under gas conditions. In this work, we developed a home-made Micro-Electrical-Mechanical-System (MEMS) heater with unprecedented small temperature gradient and thermal drift, which not only enables the temperature evolution caused by gas injection to be measured in real-time, but also made the key heat dissipation path easier to be modeled to theoretically understood and predict the temperature decrease. A new parameter termed as 'gas cooling ability $(H)$ ', determined purely by the physical properties of the gas, can be used to compare and predict the gas-induced temperature decrease by different gases. Our findings can act as a reference for predicting the real temperature for in situ heating experiments without closed-loop temperature sensing capabilities in the gas environment as well as all gas-related heating systems.
\end{abstract}

\title{
Keywords
}

Environmental TEM (ETEM), MEMS heater, in situ heating, gas cooling effect, temperature measurement 


\section{Introduction}

In situ heating experiments inside an electron microscope such as transmission electron microscope (TEM) have been widely used to study dynamic processes of temperature-induced structural transitions, such as phase transformation, melting/sublimation(Asoro, et al., 2013; Li, et al., 2019), high-temperature degradation(Divitini, et al., 2016; Wang, et al., 2020b) and precipitation(Chen, et al., 2006; Liu, et al., 2017). In recent years, the rapid development of environmental TEM (ETEM) has brought in more possibilities for in situ heating experiments, especially in gas-solid reaction related fields such as catalyst reaction(Baldi, et al., 2014; Behrens, et al., 2012; Chi, et al., 2020; Hansen, et al., 2002; Hofmann, et al., 2007; Panciera, et al., 2015; Simonsen, et al., 2010; Vendelbo, et al., 2014), nanostructure growth(Hudak, et al., 2014; Kodambaka, et al., 2007; Panciera, et al., 2015; Rackauskas, et al., 2014; Sharma \& Iqbal, 2004), and corrosion(Curnan, et al., 2019; Li, et al., 2021; Luo, et al., 2018; Wang, et al., 2020a; Zhou, et al., 2012; Zou, et al., 2017; Zou, et al., 2018). Accurate control and measurement of the real sample temperature under experiment conditions are critical for the understanding and interpretation of the experimental results. However, despite developments in closed-loop temperature-controlled micro-fabricated(MEMS) heaters, open-loop temperature-controlled heaters are still widely used in most commercial in situ TEM heating devices - including the most widely used furnace heating holders(Butler, 1979), spiral coil heating devices(Kamino, et al., 2005a; Kamino, et al., 2005b; Takeo, et al., 2006), and the most recent micro-fabricated (MEMS) heaters(Allard, et al., 2009; Allard, et al., 2012; Mele, et al., 2016) - due to their broader availability, easier sample preparation, lower cost, and broader sample compatibility with other characterization instruments.

The open-loop temperature-controlled heaters use a heating current vs. temperature curve which is pre-calibrated in the vacuum to infer the temperature from the applied current(Allard, et al., 
2009; Saka, et al., 2011). For conventional TEM studies in vacuum, this method works well. But for ETEM applications, extra power will be consumed by heat convection of the injected gas, which will lead to a significant temperature drop under constant heating current/power. For example, it was reported that under $140 \mathrm{~Pa} \mathrm{H}_{2}$, the real sample temperature in a furnace holder dropped from $500{ }^{\circ} \mathrm{C}$ to only $175^{\circ} \mathrm{C}$ (Winterstein, et al., 2015). Moreover, the real temperature is known to be affected by the experimental parameters such as gas species and gas pressure, making it unrealistic to calibrate the current-temperature curve under every condition. Consequently, a mechanistic understanding of the real temperature change under gas condition is essential for predicting and controlling the temperature.

Measuring real-time temperature inside TEM, especially in the gas environment, is a very challenging task. Although MEMS devices that have closed-loop temperature measurement capabilities(Mele, et al., 2016; van Huis, et al., 2009; van Omme, et al., 2018) have emerged recently, limited by their intrinsic structure design, these commercially available MEMS devices suffer a large temperature gradient in the sample area (up to 30\% from the hottest spot(Niekiel, et al., 2017)). Moreover, under the gas environment, the temperature distribution is changed, making it more difficult to investigate and model the real-temperature change caused by gas. Many other methods have been also developed, including measuring the lattice spacing changes in diffraction pattern (Niekiel, et al., 2017; Winterstein, et al., 2015), using the gas pressure change measured from EELS spectrum in closed gas cells (Vendelbo, et al., 2013), using size-dependent sublimation temperature of nanoparticles to calibrate local temperature(Vijayan \& Aindow, 2019), or even modifying the TEM to add a laser probe to capture local Raman spectroscopy(Picher, et al., 2015). However, they usually suffer from relatively poor temperature accuracy and lacks real-time temperature sensing capability during in situ experiments. 
In this work, we report a novel home-made MEMS heating device (named as CAMP-Nano heater) that could not only accurately measure and control the real-temperature under gas, but also significantly improve the thermal stability of the image and the temperature uniformity. We further demonstrate how gas type, gas pressure, and the set temperature affect the sample temperature. Because of the special structure design of the CAMP-Nano heater, the key heat dissipation path by the injected gas, i.e. convection, can be modeled to predict the temperature decrease. Surprisingly, we find that a new parameter termed as 'gas cooling ability $(\mathrm{H})$ ', determined purely by the physical properties of the gas under ambient conditions, can be used to predict the relative temperature decrease by different gases inside ETEM.

\section{Material and methods}

\section{The CAMP-Nano heater}

In this work, we developed a MEMS-based in situ TEM heating chip that solved the aforementioned limitations of existing heaters for accurate real-time temperature control and sensing. Figure 1(b) illustrates the core part of the CAMP-Nano heater (for more details, see (Li, et al., 2018; Li, et al., 2017)). It has a specially designed free-standing hotplate that is connected to the rest of the chip only via four springs, which makes the hotplate thermally isolated from the rest parts of the chip, and thus leads to a very uniform temperature distribution of the hotplate that is ideal for temperature sensing shown in Figure 1(c). The hotplate contains several posts for mounting samples transferred using focused ion beam (FIB). The free-standing design of the posts enables further sample thinning on the chip after FIB transfer. Besides uniform temperature distribution, this structure also solved the long-lasting z-direction sample drift problem caused by the bulging of the heating membrane, leads to significantly improved image 
stability even during temperature ramping, as shown in Figure 1(d). Platinum coil, which has a linear temperature coefficient of resistance and has been widely used for commercial resistance temperature detectors(RTDs) (Childs, et al., 2000) was employed to heat the hotplate and sense the temperature. The resistance of the heating\& sensing coil is measured via the four-terminal sensing method to ensure only the resistance on the hotplate is measured while other cable/wiring/contact resistances are counterbalanced and ruled out to make the measurement more accurate. Unlike conventional TEM heating devices that use vacuum-calibrated currenttemperature curve to infer the temperature from the applied current, the hotplate temperature in our heater is calculated from the real-time measured resistance via the pre-calibrated temperature coefficient of resistance that is not affected by the gas condition. Hence, our heater can be used to sense the sample temperature change under gas conditions. CAMP-Nano heater has a home-made control software that can easily switch on or off the close-loop feedback control even during the heating experiment, hence the heater could easily switch between the closed-loop control mode and the more widely used open-loop temperature control mode. When the feedback is turned off, the heating current is maintained constant, mimicking the open-loop heating function used in the commercial heating devices. In the open-loop mode, the temperature sensing traces on the hotplate work as temperature sensors to monitor the real-time temperature of the hotplate.

\section{Experimental setup}

Figure 1(a) shows the schematic illustration of the experiment setup. A differentially pumped ETEM (Hitachi H-9500 with a home-made gas delivery system) was used to control the gas environment. Gases were injected into the specimen chamber through a needle valve, while the gas pressure of the injected gas was measured by a vacuum gauge. The CAMP-Nano heater was used to measure the temperature changing during gas injection. 
The CAMP-Nano heater was first heated up to the set temperature ( $\left.T_{\text {set }}\right)$ using closed-loop temperature control with feedback function turned on in the vacuum. When the temperature reaches the set temperature, the feedback function was turned off so that the heating current was maintained constant while the temperature sensor continued to measure the real-time temperature. Gases were then let in through the needle valve to fill up the specimen chamber. The CAMP-Nano heater was located at the center of the specimen chamber, which is a few centimeters away from the gas injection needle, so the gas concentration and flow near the heating area can be considered uniform and stable.

The power of the real-time sensing capability and the feedback control function of the CAMPNano heater is demonstrated in Figure 1(e). When feedback control is turned off, the heating current is maintained at a constant value, similar to the open-loop temperature control used in conventional TEM heating devices. Using the temperature sensor, the real-time temperature change during gas injection is detected. When hydrogen was injected with gradually increased gas pressure up to $2 \mathrm{~Pa}$, the real-time temperature quickly dropped from the set temperature $\left(T_{\text {see }}=200^{\circ} \mathrm{C}\right.$ ) and then gradually leveled off to $\sim 176^{\circ} \mathrm{C}$ in a few minutes. When the gas was turned off, the temperature increased fast and then gradually back up to the set temperature while the chamber was gradually pumped down to vacuum (base pressure of $\sim 5 \times 10^{-4} \mathrm{~Pa}$ ). In comparison, when feedback control is turned on, during gas injection, the heating current quickly increased to compensate for the heat taken away by the gas, leaving the temperature constant throughout gasinjection. This result demonstrates the significant impact of gas injection on the sample temperature and the necessity of closed-loop temperature control for gas involved heating experiments. 


\section{Measurement of gas-induced temperature change}

To systematically understand the gas-induced sample temperature changes inside ETEM, controlled experiments with three variables are performed, i.e. set temperature $\left(T_{\text {set }}\right)$, gas pressure (Pgas), and gas type. The $T_{\text {set }}$ tested in this work is $100^{\circ} \mathrm{C}, 200^{\circ} \mathrm{C}, 300^{\circ} \mathrm{C}$, and $400{ }^{\circ} \mathrm{C}$, respectively. The $P_{\text {gas }}$ programmed in this work are 1, 2, 3, 4, $5 \mathrm{~Pa}$ for each gas species, measured by a vacuum gauge near the heating device inside the specimen chamber. The gas species explored in this work are $\mathrm{H}_{2}, \mathrm{~N}_{2}, \mathrm{O}_{2}$, and $\mathrm{CO}_{2}$, all with ultra-high purity (99.999\%). Each time when the injected gas pressure increased to the setpoint, the pressure was kept for more than 100 seconds for the gas to be uniformly distributed inside the chamber as well as for the temperature to settle. When switching gas types, the gas injection pipeline and the specimen chamber were purged several times with the aim gas and the gas concentration was confirmed by a residual gas analyzer attached to the pumping lines of the specimen chamber. To rule out the temperature contribution from the electron beam illumination(Kritzinger \& Ronander, 1974; Thornburg \& Wayman, 1973), the electron beam was turned off during the measurement. All temperature curves were recorded through the home-made control software.

\section{Results \& Discussion}

\section{Theoretical analysis of the gas-induced heat dissipation inside ETEM}

Since the hotplate of the CAMP-Nano heater is thermally isolated from the rest parts, the heat dissipation of the hotplate can be simplified as a flat plate, as schematically illustrated in Figure 2, which is widely used in heat transfer theories (Bergman, et al., 2011; Kreith, et al., 2012; Springer, 1971; Sundén $\& \mathrm{Fu}, 2017 \mathrm{a})$. The total input power $\Phi_{\text {in }}$ of the hotplate came from the Joule heating of the heating elements, hence $\Phi_{i n}=I^{2} R$. As shown in Figure 2(a), under thermal equilibrium 
condition with injected gas, $\Phi_{\text {in }}=\Phi_{c o n d}+\Phi_{\text {rad }}+\Phi_{c o n v}$. Where $\Phi_{c o n d}$ is the dissipated by heat conduction to the rest parts of the heater through the 4 springs, $\Phi_{r a d}$ is the power consumed by thermal radiation and $\Phi_{c o n v}$ is the power consumed by heat convection of the injected gas. In this work, we take $\Phi_{\text {cond }}$ and $\Phi_{\text {rad }}$ as constant for the given testing conditions and mainly consider the gas-induced temperature variation $\Phi_{c o n v}$ and its affecting factors.

The extra heat convection taken away by the injected gas can be simplified as the heat convection on a flat plate model illustrated in Figure 2(b). After gas injection, since the input heating current remains constant, the input power is insufficient to supply the increased power consumption, hence the hotplate temperature $T_{h}$ dropped from the original set temperature $T_{\text {set }}$ to the settled temperature under gas $T_{\text {read. }}$. Meanwhile, the gas temperature $T_{g}$ increased from ambient temperature $T_{0}$ (in this case, $T_{0}=$ room temperature) to a gradient on the hotplate surface boundary layer shown by the blue shaped area in Figure 2(b), in which the gas in contact with the plate surface is heated up to $T s$.

To get the function of $\Phi_{c o n v}$ in the flat plate heat convection model, the gas flow condition is required. Under our experiment condition $\left(P=1 \sim 5 \mathrm{~Pa}, T_{\text {gas }}=20^{\circ} \mathrm{C}\right)$, the mean free path of the gas is at thousand-micrometer scale, while the dimension of the gas chamber is at $\sim 30 \mathrm{~cm}$ scale, leading to a Knudsen number $(K n)$ between $0.001 \sim 0.1$, indicating a flow condition between slip flow and continuum flow. (Springer, 1971; Sundén \& Fu, 2017b)As shown in Figure 2(c), for high gas pressure range ( $P>3 \mathrm{~Pa}$ for most gases), the gas flow in ETEM can be considered continuum flow, in this case, the gas temperature at the surface is the same as the heater temperature, i.e. $T_{s}=T_{\text {set. }}$. While for medium gas pressure range $(0.2 \mathrm{~Pa} \sim 3 \mathrm{~Pa})$, the gas flow follows slip flow, in this case, there is a temperature jump between the surface and the adjacent gas, i.e. $T_{s}=T_{s e t}-T_{j}$, where $T_{j}$ is the temperature jump that depends on the gas and the surface condition(Springer, 1971; Sundén \& 
Fu, 2017a). Nevertheless, the heat transfer equations for continuum flow can still be used. For lower gas pressure range, transitional flow $\left(10^{-2} \mathrm{~Pa} \sim 0.1 \mathrm{~Pa}\right)$ or free molecular flow $\left(\mathrm{P}<10^{-2} \mathrm{~Pa}\right)$ dominates, the heat transfer function is different. Moreover, for MEMS gas cells, the gas flow condition is also different due to the much smaller characteristic length for gas flow. Luckily, the gas-induced temperature change in the low gas pressure range is neglectable, and MEMS gas cells usually operate under very high pressure that obeys continuum flow and contains close-loop temperature sensing to accommodate the gas-induced temperature change. Hence, our analysis should cover most applications for gas-induced temperature change in differentially-pumped ETEM.

In the continuum flow range, the gas flow speed is needed to distinguish whether the gas flow follows laminar flow or turbulence flow. In a similar experiment inside ETEM, Winterstein et al (Winterstein, et al., 2015) reported a calculated Reynolds number of 0.362 under 135 Pa gas pressure, indicating laminar flow. Since the maximum gas pressure used in this work is only $5 \mathrm{~Pa}$, the gas flow should also lay in the laminar flow regime. Hence the heat convection can be calculated as (Bergman, et al., 2011; Kreith, et al., 2012):

$$
\Phi_{\text {conv }}=\frac{\kappa A\left(T_{S}-T_{0}\right)}{L} \cdot 0.664 \cdot \sqrt[3]{\operatorname{Pr}} \cdot \sqrt{R e}
$$

Where $\operatorname{Pr}$ is the Prandtl number, $R e$ is the Reynolds number, both numbers are determined by the gas parameters, $L$ is the length of the hotplate, $A$ is the surface area of the hotplate, $\kappa$ is the thermal conductivity of the gas, $T_{0}$ and $T_{s}$ are the temperatures of the injected gas before and after convection shown in Figure 2(b). By simplifying this equation, we can get:

$$
\Phi_{\text {conv }}=0.664 \cdot \sqrt{u} \cdot \Delta T \cdot \frac{A}{\sqrt{L}} \cdot \frac{\kappa^{\frac{2}{3}} \cdot \frac{1}{3} \cdot \rho^{\frac{1}{2}}}{\mu^{\frac{1}{6}}}
$$


where $\Delta T=T_{S}-T_{0}$ determined by the temperature difference between the hotplate and the gas, $\frac{A}{\sqrt{L}}$ is determined by the geometry of the hotplate, $u$ is the flow speed of the gas determined by the gas pressure(Lafferty, 2003), while the last item of the function is determined purely by gas properties: $\kappa$-thermal conductivity, $\mathrm{c}_{\mathrm{p}}$-thermal capacity at constant pressure, $\rho$-gas density, $\mu$ dynamic viscosity.

In this work, we define:

$$
H=\frac{\kappa^{\frac{2}{3}} \cdot c_{p}^{\frac{1}{3}} \cdot \rho^{\frac{1}{2}}}{\mu^{\frac{1}{6}}}
$$

Hence, equation (2) can be simplified as:

$$
\Phi_{\text {conv }} \propto \sqrt{u} \cdot \Delta T \cdot \frac{A}{\sqrt{L}} \cdot H
$$

For slip flow, $\Delta T=T_{\text {set }}-T_{0}-T_{j}$, where $T_{j}$ is the temperature jump that depends on the gas and the surface(Springer, 1971; Sundén \& Fu, 2017b):

$$
T_{j}=\frac{2-\alpha}{\alpha} \frac{2 \gamma}{\gamma+1} \cdot \frac{\lambda}{P r} \cdot \frac{\partial T_{g}}{\partial d}
$$

Where $\alpha$ is the gas accommodation coefficient that depends on the gas type and surface material and condition, $\lambda$ is the mean free path of the gas that depends on gas type, pressure, and temperature.

Based on equation (4), the key factors that affect gas-induced heat dissipation inside ETEM can be summarized as below:

1) Gas pressure effect: the larger gas flow speed $u$, the higher gas pressure $P_{\text {gas. }}$ Therefore, higher gas pressure is expected to cause a larger temperature drop for given testing 
conditions.

2) Set temperature effect: the larger $\Delta T$ between the gas and hotplate, the more power will be consumed by heat convection $\Phi_{c o n v}$, hence a larger temperature drop is expected.

Given fixed $T_{0}$, as used in our experiment, a higher $T_{\text {set }}$ means a larger temperature drop.

3) Heater geometry effect: the larger the heating area $A$, the more power consumption by heat convection $\Phi_{c o n v}$ and larger temperature drop will be expected. Because the surface area of a traditional furnace heater is usually $\sim 100$ times larger than a MEMS heater, the temperature drop for a conventional furnace heater is expected to be much larger than the MEMS heaters for given testing conditions.

4) Gas species effect: Gases with larger $H$ will cause more temperature drop.

Next, we will demonstrate the equation 4 we deduced is at least quantitatively correct to predict the gas-induced temperature evolution.

\section{Effect of gas pressure and set temperature}

Figure 3(a) shows a typical measured temperature curve with stepwise increased $\mathrm{H}_{2}$ gas pressure. The hotplate was heated to a set temperature $T_{\text {set }}$ of $400{ }^{\circ} \mathrm{C}$ in the vacuum, then the feedback control function was turned off in the home-made control software to maintain a constant heating current. At $\sim 60 \mathrm{~s}, \mathrm{H}_{2}$ gas was injected with a stepwise profile up to $5 \mathrm{~Pa}$ with steps of $1 \mathrm{~Pa}$ and the gas pressure was kept constant for $\sim 60 \mathrm{~s}$ at each step. As can be noticed from the plot, the temperature readout changes simultaneously with the pressure change, and is quite stable at each constant step period. When the gas was turned off at $\sim 300$ s, the temperature returns to the initial set temperature together with the pumping down of the chamber vacuum. Obviously, higher pressure led to a larger temperature drop, as predicted by equation 4 . 
In order to better compare the temperature drop at different set temperatures, we define another parameter, i.e. the normalized temperature $T_{N}$ as:

$$
T_{N}=\frac{T_{\text {read }}-T_{0}}{T_{\text {set }}-T_{0}}
$$

Where $T_{\text {read }}$ represents real-time temperature. $T_{N}$ can be understood as the ratio of the temperature difference between heater and gas ( $\Delta T$ in equation (4)) after and before gas injection. For example, when $T_{\text {set }}=400{ }^{\circ} \mathrm{C}$, the real temperature dropped to $333.9{ }^{\circ} \mathrm{C}$ under $5 \mathrm{~Pa} \mathrm{H}$, so the normalized temperature $T_{\mathrm{N}}=82.6 \%$, indicating the relative temperature difference between the heater and the gas after gas injection is $82.6 \%$ of the value before gas injection.

Using this normalized temperature, the temperature drop with increasing gas pressure under different set temperature can be directly compared, as plotted in Figure 3(b). Each curve shows the evolution of the normalized temperature in response to the pressure increase under the same set temperature. As shown in Figure 3(b), for a given set temperature, the normalized temperature decreases with increasing gas pressure. The higher the set temperature, the larger the normalized temperature drop. Again, the observed phenomena agree well with Equation 4.

It is worth noting that curves shown in Figure 3(b) are gradually deviating from linear fitting along with the increase of the set temperature. This can be rationalized as below: the power consumed by heat convection $\Phi_{\text {conv }}$ is proportional to the square root of the gas flow speed $u, \Phi_{\text {conv }} \propto \sqrt{u}$. The steady-state gas flow speed is determined by the gas pressure and ETEM pumping speed, which increases with increasing gas pressure and gradually decrease the increase rate until a maximum speed is reached (Lafferty, 2003). So the gas flow speed is expected to increase with increasing gas pressure, and the speed will gradually approach a constant value determined by the maximum pumping speed of the ETEM. Hence the normalized temperature is expected to decrease 
non-linearly with increasing gas pressure.

Similarly, the effect of set temperature under given gas pressures are plotted in Figure 3(c), each curve shows the normalized temperature change in response to increasing set temperature. Under the same gas pressure, the higher the set temperature, the larger the normalized temperature drop. The $T_{N}$ decreases linearly with increasing set temperature, which meets the prediction by equation 4. Using equation (6), the read temperature decreases parabolically with increasing set temperature.

\section{Effect of heater geometry}

Although only the CAMP-Nano heater was used in this work, the prediction on the heating area can be verified by other literature reports. Winterstein et al. (Winterstein, et al., 2015) measured the temperature change of furnace heater and MEMS heater using the lattice parameter change in the diffraction pattern of $\mathrm{Ag}$ nanoparticles, and found under $140 \mathrm{~Pa} \mathrm{H}_{2}$, the temperature of furnace heater dropped from $500{ }^{\circ} \mathrm{C}$ to $\sim 175^{\circ} \mathrm{C}$, while the MEMS heater only dropped from $400{ }^{\circ} \mathrm{C}$ to $\sim 220^{\circ} \mathrm{C}$.

\section{Effect of gas species}

We found that under a given set temperature and pressure, different gases can cause very different temperature drop, as summarized in Figure 4. Four different gases were tested in our work, including $\mathrm{H}_{2}, \mathrm{~N}_{2}, \mathrm{O}_{2}$, and $\mathrm{CO}_{2}$. As plotted in Figure 4(a), under the same set temperature $T_{\text {set }}=$ $400{ }^{\circ} \mathrm{C}$, the normalized temperature drops with increasing gas pressure $P_{\text {gas }}$ for all tested gases, and the relative decrease amount ranks in the order of $\mathrm{H}_{2}>\mathrm{O}_{2} \approx \mathrm{N}_{2}>\mathrm{CO}_{2}$ at every tested gas pressure. Similarly, as plotted in Figure 4(b), under the same gas pressure $P_{\text {gas }}=5 \mathrm{~Pa}$, the normalized temperature also drops linearly with increasing set temperature $T_{\text {set }}$, and the relative decrease amount ranks in the same order of $\mathrm{H}_{2}>\mathrm{O}_{2} \approx \mathrm{N}_{2}>\mathrm{CO}_{2}$ at every tested set temperature. When 
$T_{\text {set }}=400{ }^{\circ} \mathrm{C}, P_{\text {gas }}=5 \mathrm{~Pa}$, the normalized temperature drop $\Delta T_{N}$ caused by $\mathrm{H}_{2}, \mathrm{O}_{2}, \mathrm{~N}_{2}$, and $\mathrm{CO}_{2}$ are $17.4 \%, 7.9 \%, 7.9 \%$, and $6.2 \%$ respectively, i.e. the real temperature drops by $66.1{ }^{\circ} \mathrm{C}, 29.9^{\circ} \mathrm{C}$, $29.8^{\circ} \mathrm{C}$, and $23.4^{\circ} \mathrm{C}$ respectively.

In order to know the relative cooling ability of different gases in a more intuitive manner, we define relative normalized temperature drop $\Delta T_{R N}=\Delta T_{N, \mathrm{gas}} / \Delta T_{N, \mathrm{H} 2}$. As shown in Figure 5(a), for $T_{\text {set }}=$ $400{ }^{\circ} \mathrm{C}$, the $\Delta T_{R N} v s$. gas species curves are similar to each other with the $\Delta T_{R N}$ increasing with increasing gas pressure for the same gas. The difference in the absolute value for each gas might stem from the justification from the slip flow of the gas under lower pressure. While for $P_{\text {gas }}=5$ $\mathrm{Pa}$, as shown in Figure 5(b), the $\Delta T_{R N}$ almost remains unchanged for each gas for the $T_{\text {set }}$ ranged from $100{ }^{\circ} \mathrm{C}$ to $400{ }^{\circ} \mathrm{C}$. This inspired us to consider that the relative cooling ability is a more physical properties dependent parameter and therefore should be linked with $\mathrm{H}$, as we defined in equation (3). Using the physical properties of the gases shown in Table 1, the value of $H$ for different gases can be calculated. Similarly, we define relative gas cooling ability $H_{R}=H_{g a s} / H_{H 2}$. Surprisingly, the trend of $H_{R} v s$. gas species is very similar to that of $\Delta T_{R N}$, as shown in Fig. 5c. It is worth noting that all the parameters listed in Table 1 are measured under ambient temperature and pressure, i.e. $20^{\circ} \mathrm{C}$ and $1 \mathrm{~atm}$, the values might change under different temperature and pressure, which might be the reason for the deviation between the calculated value in Figure 5(c) and experimental value in Figure 5(a-b). Nevertheless, the relative ratio among different gases meets well with the experimental results. Therefore, it can be concluded that $H$ can be used as a scale to reflect the cooling ability among different gases, regardless of pressure and temperature. Consequently, we term $H$ as the gas cooling ability. Our findings were further supported by the following facts: by calculating the $H$ of commonly used gas species as listed in Table 1 and plot them all together in Figure 6, the following gas cooling trend can be inferred: $\mathrm{H}_{2}>\mathrm{He}>\mathrm{Air}>\mathrm{O}_{2} \approx$ 
$\mathrm{N}_{2} \approx \mathrm{CO}>\mathrm{CO}_{2}>$ Ar. This meets well with previous literature reports that $\mathrm{H}_{2}$ and $\mathrm{He}$ cause the most significant temperature decrease.(Picher, et al., 2015)

\section{Conclusion}

In summary, in this work, a novel home-made MEMS heating device with a thermally isolated hotplate and real-time temperature sensing capability was developed, which enables accurate real-time temperature measurement and simplifies the theoretical modeling to quantify the gas effect on the sample temperature during in situ heating experiments in environmental TEM. Our theoretical modeling deduced the equation to calculate the power consumed by the injected gas to predict the temperature change. A new parameter, gas cooling ability $H$, which is determined purely by some physical parameters of the gas, is defined to predict the temperature decrease under different gas species. Our results show that the real temperature of the heating devices is very sensitive to the gas environment, for example, even $5 \mathrm{~Pa}$ of $\mathrm{H}_{2}$ can cause the temperature to drop from $400{ }^{\circ} \mathrm{C}$ to $333^{\circ} \mathrm{C}$. Hence, for in situ heating experiments under the gas environment, real-time temperature sensing and closed-loop temperature control are essential for accurate temperature. However, open-loop heating devices are still widely used. For open-loop heating devices, the real temperature under gas needs to be compensated. The temperature drop caused by gas injection is determined by gas type, gas pressure, heater temperature $T_{\text {set }}$, and heating area $A$ of the heating device. Our results indicate the normalized temperature drop $\Delta T_{N}$ increases nonlinearly with increasing gas pressure $P_{\text {gas }}$ and linearly with increasing initial heater temperature $T_{\text {set. }}$ And among the tested gas species, the gases follow the order of $\mathrm{H}_{2}>\mathrm{O}_{2} \approx \mathrm{N}_{2}>\mathrm{CO}_{2}$. Using the calculated $H$, the temperature drop caused by different gases can be predicted in the order of $\mathrm{H}_{2}>\mathrm{He}>\mathrm{Air}>\mathrm{O}_{2} \approx \mathrm{N}_{2} \approx \mathrm{CO}>\mathrm{CO}_{2}>$ Ar. These results can act as a reference to predict the real temperature of in situ TEM heating experiments in the gas 
environment to better understand and explain the experimental observations. These results would also be helpful for all gas-related heating systems.

\section{Acknowledgments}

This work is supported by the National Key Research and Development Program of China (No. 2017YFB0702001) and National Natural Science Foundation of China (grant numbers NSFC 5203000210, 51621063), National Science Foundation (grant numbers NSF DMR-1508417, CMMI-1905647) and the International Joint Laboratory for Micro/Nano Manufacturing and Measurement Technologies. The MEMS fabrications were performed at King Abdullah University of Science and Technology (KAUST). We acknowledge Dr. Longqing Chen, Ms. Chunxiu Tian,

Dr. Qiang Zhang from KAUST for the help in MEMS design and fabrication, Ms. Bo Fu from

Xi'an Jiaotong University (XJTU) for the helpful discussions in the theory part, Ms. Qinqin Fu from XJTU and Hitachi High Technologies for the technical supports.

\section{References}

Allard, L.F., Bigelow, W.C., Jose-Yacaman, M., Nackashi, D.P., Damiano, J. \& Mick, S.E. (2009). A new MEMS-based system for ultra-high-resolution imaging at elevated temperatures. Microscopy research and technique 72(3), 208-215.

Allard, L.F., Overbury, S.H., Bigelow, W.C., Katz, M.B., NACKashi, D.P. \& Damiano, J. (2012). Novel MEMS-based gas-cell/heating specimen holder provides advanced imaging capabilities for in situ reaction studies. Microsc Microanal 18(4), 656-666.

Asoro, M.A., Kovar, D. \& FerreIRA, P.J. (2013). In situ transmission electron microscopy observations of sublimation in silver nanoparticles. ACS nano 7(9), 7844-7852.

BAldi, A., NARAYAn, T.C., KoH, A.L. \& Dionne, J.A. (2014). In situ detection of hydrogeninduced phase transitions in individual palladium nanocrystals. Nature materials 13(12), 1143-1148.

Behrens, M., Studt, F., Kasatkin, I., Kuhl, S., Havecker, M., Abild-Pedersen, F., Zander, S., Girgsdies, F., Kurr, P., KnieP, B.L., Tovar, M., Fischer, R.W., Norskov, J.K. \& SchlOGL, R. (2012). The active site of methanol synthesis over $\mathrm{Cu} / \mathrm{ZnO} / \mathrm{Al} 2 \mathrm{O} 3$ industrial catalysts. Science 336(6083), 893-897.

Bergman, T.L., InCROPERA, F.P., DEWITT, D.P. \& LAVINE, A.S. (2011). Fundamentals of heat and mass transfer. John Wiley \& Sons. 
BUTLER, E.P. (1979). In situ experiments in the transmission electron microscope. Reports on Progress in Physics 42(5), 833-895.

Chen, J.H., Costan, E., van Huis, M.A., Xu, Q. \& Zandbergen, H.W. (2006). Atomic pillarbased nanoprecipitates strengthen AlMgSi alloys. Science 312(5772), 416-419.

Chi, H., Curnan, M.T., Li, M., Andolina, C.M., SAidi, W.A., Veser, G. \& YAnG, J.C. (2020). In situ environmental TEM observation of two-stage shrinking of $\mathrm{Cu} 2 \mathrm{O}$ islands on $\mathrm{Cu}(100)$ during methanol reduction. Physical Chemistry Chemical Physics 22(5), 27382742.

Childs, P.R.N., GreEnwoOd, J.R. \& LONG, C.A. (2000). Review of temperature measurement. Review of Scientific Instruments 71(8), 2959-2978.

Curnan, M.T., Andolina, C.M., Li, M., Zhu, Q., Chi, H., SAidi, W.A. \& YAnG, J.C. (2019). Connecting Oxide Nucleation and Growth to Oxygen Diffusion Energetics on Stepped $\mathrm{Cu}(011)$ Surfaces: An Experimental and Theoretical Study. Journal of Physical Chemistry C 123(1), 452-463.

Divitini, G., Cacovich, S., Matteocci, F., CinÀ, L., Di Carlo, A. \& DuCATi, C. (2016). In situ observation of heat-induced degradation of perovskite solar cells. Nature Energy 1(2).

Hansen, P.L., Wagner, J.B., Helveg, S., Rostrup-Nielsen, J.R., Clausen, B.S. \& Topsoe, H. (2002). Atom-resolved imaging of dynamic shape changes in supported copper nanocrystals. Science 295(5562), 2053-2055.

Hofmann, S., Sharma, R., Ducati, C., Du, G., Mattevi, C., Cepek, C., Cantoro, M., Pisana, S., Parvez, A., Cervantes-Sodi, F., Ferrari, A.C., Dunin-Borkowski, R., Lizzit, S., PETACCIA, L., GOLDONI, A. \& ROBERTSON, J. (2007). In situ observations of catalyst dynamics during surface-bound carbon nanotube nucleation. Nano Lett 7(3), 602-608.

Hudak, B.M., Chang, Y.J., Yu, L., Li, G., Edwards, D.N. \& Guiton, B.S. (2014). Real-time observation of the solid-liquid-vapor dissolution of individual tin(IV) oxide nanowires. ACS nano 8(6), 5441-5448.

Kamino, T., Yaguchi, T., Konno, M., Watabe, A., Marukawa, T., Mima, T., Kuroda, K., SAKA, H., Arai, S., MaKino, H., SuZUKI, Y. \& KishitA, K. (2005a). Development of a gas injection/specimen heating holder for use with transmission electron microscope. Journal of electron microscopy 54(6), 497-503.

Kamino, T., Yaguchi, T., SAto, T. \& Hashimoto, T. (2005b). Development of a technique for high resolution electron microscopic observation of nano-materials at elevated temperatures. Journal of electron microscopy 54(6), 505-508.

KodAmbaka, S., Tersoff, J., Reuter, M.C. \& Ross, F.M. (2007). Germanium nanowire growth below the eutectic temperature. Science 316(5825), 729-732.

Kreith, F., Manglik, R.M. \& Bohn, M.S. (2012). Principles of heat transfer. Cengage learning.

KRITZINGER, S. \& RONANDER, E. (1974). Local beam heating in metallic electron microscope specimens. Journal of Microscopy 102(2), 117-124.

LAFFERTY, J.M. (2003). Vacuum Technology. In Encyclopedia of Physical Science and Technology, pp. 385-410. Elsevier.

Li, J., WANG, Z., LI, Y. \& DEEPAK, F.L. (2019). In Situ Atomic-Scale Observation of Kinetic Pathways of Sublimation in Silver Nanoparticles. Advanced Science 6(8).

Li, M., Curnan, M.T., Gresh-Sill, M.A., House, S.D., SAidi, W.A. \& YanG, J.C. (2021). Unusual layer-by-layer growth of epitaxial oxide islands during $\mathrm{Cu}$ oxidation. Nature Communications 12(1), 2781.

LI, M., XIE, D.-G. \& SHAN, Z.-W. (2018). Development of a Novel Bulk Sample TEM Heater and 
Its Application. Microscopy and Microanalysis 24(S1), 1830-1831.

LI, M., XIE, D.G., MA, E., LI, J., ZHANG, X.X. \& SHAN, Z.W. (2017). Effect of hydrogen on the integrity of aluminium-oxide interface at elevated temperatures. Nat Commun 8, 14564.

Liu, C., Malladi, S.K., Xu, Q., Chen, J., TichelaAr, F.D., Zhuge, X. \& Zandbergen, H.W. (2017). In-situ STEM imaging of growth and phase change of individual CuAIX precipitates in Al alloy. Sci Rep 7(1), 2184.

Luo, L., Su, M., Yan, P., Zou, L., Schreiber, D.K., BAer, D.R., Zhu, Z., Zhou, G., WAng, Y., BRUEMMER, S.M., XU, Z. \& WANG, C. (2018). Atomic origins of water-vapour-promoted alloy oxidation. Nature Materials, 1-6.

Mele, L., Konings, S., Dona, P., Evertz, F., Mitterbauer, C., Faber, P., Schampers, R. \& JINSCHEK, J.R. (2016). A MEMS-based heating holder for the direct imaging of simultaneous in-situ heating and biasing experiments in scanning/transmission electron microscopes. Microscopy research and technique 79(4), 239-250.

NieKiel, F., Kraschewski, S.M., Muller, J., Butz, B. \& SPIECKer, E. (2017). Local temperature measurement in TEM by parallel beam electron diffraction. Ultramicroscopy 176(August), 161-169.

Panciera, F., Chou, Y.C., Reuter, M.C., Zakharov, D., Stach, E.A., Hofmann, S. \& Ross, F.M. (2015). Synthesis of nanostructures in nanowires using sequential catalyst reactions. Nature materials 14(8), 820-825.

Picher, M., Mazzucco, S., Blankenship, S. \& Sharma, R. (2015). Vibrational and optical spectroscopies integrated with environmental transmission electron microscopy. Ultramicroscopy 150, 10-15.

Rackauskas, S., Jiang, H., Wagner, J.B., Shandakov, S.D., Hansen, T.W., Kauppinen, E.I. \& NASIBULIN, A.G. (2014). In situ study of noncatalytic metal oxide nanowire growth. Nano Lett 14(10), 5810-5813.

SAKA, H., KAMINO, T., ARA, S. \& SASAKI, K. (2011). In Situ Heating Transmission Electron Microscopy. MRS Bulletin 33(2), 93-100.

SHARMA, R. \& IQBAL, Z. (2004). In situ observations of carbon nanotube formation using environmental transmission electron microscopy. Applied Physics Letters 84(6), 990-992.

Simonsen, S.B., Chorkendorff, I., DAhl, S., Skoglundh, M., Sehested, J. \& Helveg, S. (2010). Direct observations of oxygen-induced platinum nanoparticle ripening studied by in situ TEM. J Am Chem Soc 132(23), 7968-7975.

SPRINGER, G.S. (1971). Heat Transfer in Rarefied Gases. In Advances in Heat Transfer Volume 7 , pp. 163-218.

SundÉn, B. \& Fu, J. (2017a). Aerodynamic Heating. In Heat Transfer in Aerospace Applications, pp. 27-44. Elsevier.

SundÉN, B. \& Fu, J. (2017b). Low-Density Heat Transfer. In Heat Transfer in Aerospace Applications, pp. 45-70. Elsevier.

Takeo, K., Toshie, Y., Mitsuru, K., AKIRA, W. \& Yasuhira, N. (2006). Development of a specimen heating holder with an evaporator and gas injector and its application for catalyst. Journal of electron microscopy 55(5), 245-252.

ThORNBURG, D. \& WAYMAN, C. (1973). Specimen temperature increases during transmission electron microscopy. physica status solidi (a) 15(2), 449-453.

van Huis, M.A., Young, N.P., PAndRAud, G., Creemer, J.F., VANMAeKelBerGh, D., KirKLAND, A.I. \& ZANDBERGEN, H.W. (2009). Atomic imaging of phase transitions and morphology transformations in nanocrystals. Adv Mater 21(48), 4992-4995. 
van OMme, J.T., ZakhozheVa, M., Spruit, R.G., Sholkina, M. \& Pérez Garza, H.H. (2018). Advanced microheater for in situ transmission electron microscopy; enabling unexplored analytical studies and extreme spatial stability. Ultramicroscopy 192, 14-20.

Vendelbo, S.B., Elkjaer, C.F., Falsig, H., Puspitasari, I., Dona, P., Mele, L., Morana, B., Nelissen, B.J., van RiJn, R., CREEMER, J.F., KoOYMAN, P.J. \& HELVEG, S. (2014). Visualization of oscillatory behaviour of $\mathrm{Pt}$ nanoparticles catalysing $\mathrm{CO}$ oxidation. Nature materials 13(9), 884-890.

Vendelbo, S.B., Kooyman, P.J., Creemer, J.F., Morana, B., Mele, L., Dona, P., Nelissen, B.J. \& HELVEG, S. (2013). Method for local temperature measurement in a nanoreactor for in situ high-resolution electron microscopy. Ultramicroscopy 133, 72-79.

VIJAYAN, S. \& AINDOW, M. (2019). Temperature calibration of TEM specimen heating holders by isothermal sublimation of silver nanocubes. Ultramicroscopy 196, 142-153.

WANG, Y., LI, M., YANG, Y., ZHAO, X.A., MA, E. \& SHAN, Z. (2020a). In-situ surface transformation of magnesium to protect against oxidation at elevated temperatures. Journal of Materials Science and Technology 44, 48-53.

WANG, Z., TANG, Y., ZhANG, L., LI, M., SHAN, Z. \& HuANG, J. (2020b). In Situ TEM Observations of Discharging/Charging of Solid-State Lithium-Sulfur Batteries at High Temperatures. Small 16(28), 1-8.

WintersteIn, J.P., Lin, P.A. \& SHARMA, R. (2015). Temperature Calibration for In Situ Environmental Transmission Electron Microscopy Experiments. Microsc Microanal 21(6), 1622-1628.

Zhou, G., Luo, L., Li, L., Ciston, J., STACH, E.A. \& YANG, J.C. (2012). Step-edge-induced oxide growth during the oxidation of Cu surfaces. Phys Rev Lett 109(23), 235502.

Zou, L., Li, J., ZAKHAROV, D., STACH, E.A. \& ZHOU, G. (2017). In situ atomic-scale imaging of the metal/oxide interfacial transformation. Nat Commun 8(1), 307.

Zou, L., Yang, C., Lei, Y., ZaKharov, D., Wiezorek, J.M.K., Su, D., Yin, Q., Li, J., LiU, Z., StACH, E.A., YANG, J.C., QI, L., WANG, G. \& ZHOU, G. (2018). Dislocation nucleation facilitated by atomic segregation. Nature materials 17(1), 56-63. 
Table 1|Physical properties of some typical gas species and their calculated cooling abilities

\begin{tabular}{|c|c|c|c|c|c|c|}
\hline $\begin{array}{c}\text { Gas } \\
\text { species }\end{array}$ & $\begin{array}{c}\text { Density } \\
(\rho)^{*}\end{array}$ & $\begin{array}{c}\text { Heat } \\
\text { capacity } \\
\left(\mathrm{C}_{\mathrm{p}, \mathrm{m}}\right)^{*}\end{array}$ & $\begin{array}{c}\text { Thermal } \\
\text { conductivity } \\
\text { (k)* }\end{array}$ & $\begin{array}{c}\text { Dynamic } \\
\text { viscosity } \\
(\mu)^{*}\end{array}$ & $\begin{array}{c}\text { Cooling } \\
\text { ability } \\
\text { (H) }\end{array}$ & $\begin{array}{c}\text { Relative } \\
\text { cooling } \\
\text { ability }\left(\mathbf{H}_{\mathbf{R}}\right)\end{array}$ \\
\hline & $\mathrm{kg} / \mathrm{m}^{3}$ & $\mathrm{~J} /(\mathrm{g} \cdot \mathrm{K})$ & $\mathrm{W} /(\mathrm{m} \cdot \mathrm{K})$ & $\begin{array}{l}\times 10^{-6} \\
\mathrm{~N} \cdot \mathrm{s} / \mathrm{m}^{2}\end{array}$ & & $\%$ \\
\hline $\mathbf{H}_{2}$ & 0.0899 & 14.32 & 0.168 & 8.8 & 0.1024 & 100 \\
\hline He & 0.1664 & 5.19 & 0.142 & 19.6 & 0.0777 & 75.9 \\
\hline Air & 1.205 & 1.01 & 0.0262 & 18.2 & 0.0398 & $\begin{array}{l}38.8 \\
\end{array}$ \\
\hline $\mathbf{O}_{2}$ & 1.331 & 0.919 & 0.024 & 20.4 & 0.0375 & 36.6 \\
\hline $\mathbf{N}_{2}$ & 1.165 & 1.04 & 0.024 & 18.9 & 0.0370 & 36.1 \\
\hline $\mathrm{CO}$ & 1.165 & 1.02 & 0.0232 & 17.4 & 0.0364 & 35.6 \\
\hline $\mathrm{CO}_{2}$ & 1.842 & 0.844 & 0.0146 & 14.7 & 0.0325 & 31.7 \\
\hline $\mathbf{A r}$ & 1.661 & 0.52 & 0.016 & 22.3 & 0.0260 & 25.4 \\
\hline
\end{tabular}

* parameters measured at $20^{\circ} \mathrm{C}$ and $1 \mathrm{~atm}$

Figure legends 


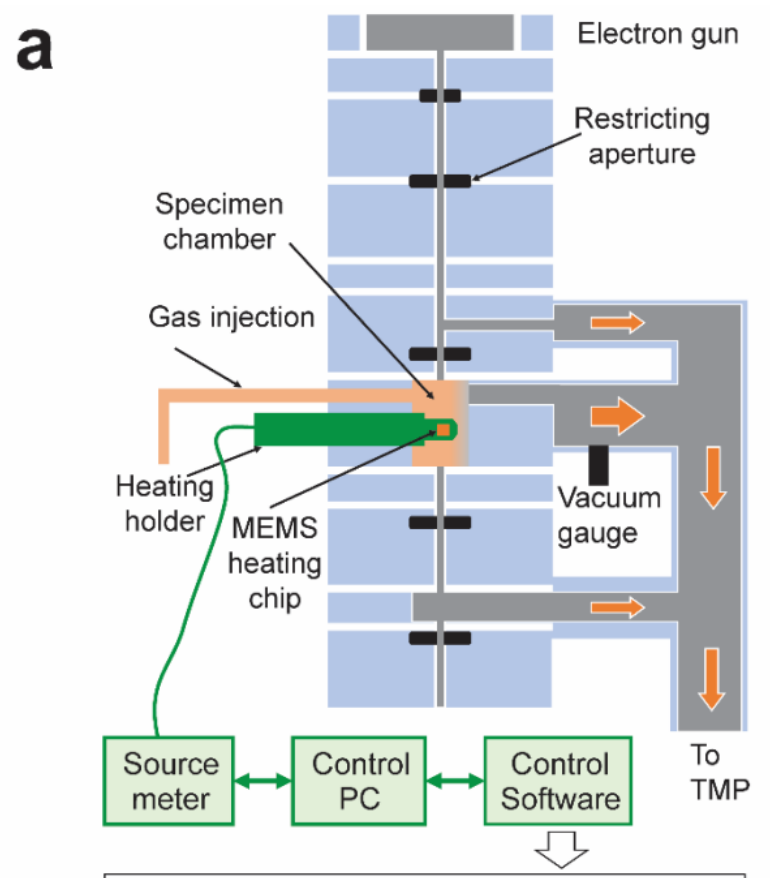

Real time temperature sensing \&control
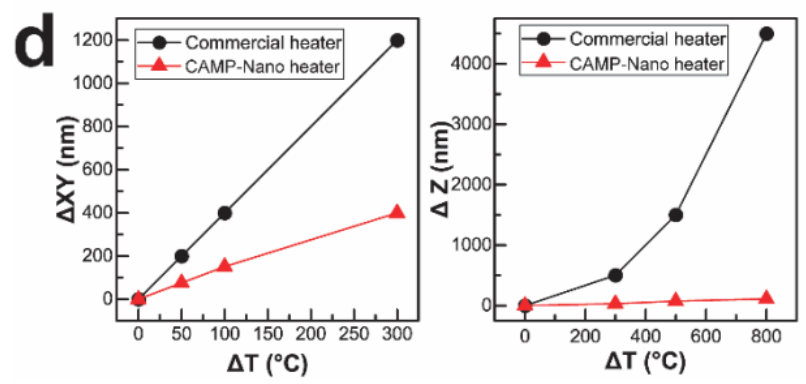
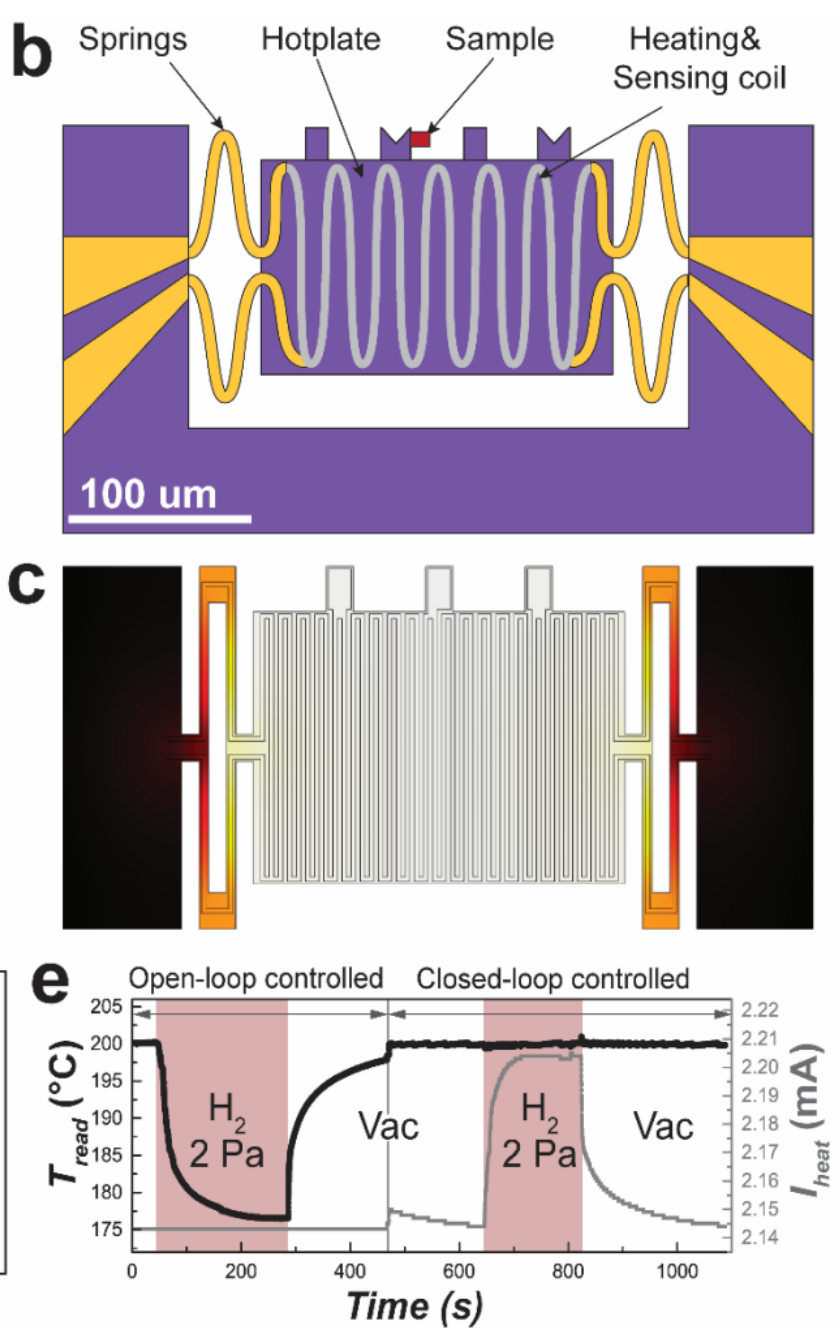

Figure 1 | Schematic illumination of the experimental setup. a) Schematic of an ETEM with CAMP-

Nano heater and temperature sensing system. b) Schematic of the core part of the CAMP-Nano heater with real-time temperature sensing capability. c) Finite Element Analysis (FEA) simulation shows very uniform temperature distribution on the hotplate (brighter color represents higher temperature) with ignorable temperature gradient, hence more accurate temperature sensing and control can be achieved. d) Drift distance of the CAMP-Nano heater (red triangles) in comparison with commercial heaters (black circles) under the same temperature jump measured inside the TEM. The CAMP-Nano heater shows much better image stability under temperature jump in both the planer direction $(\Delta \mathrm{XY})$ and the e-beam direction $(\Delta \mathrm{Z})$. e) A typical temperature curve measured during gas injection with open-loop (left) and closed-loop (right) temperature control. 

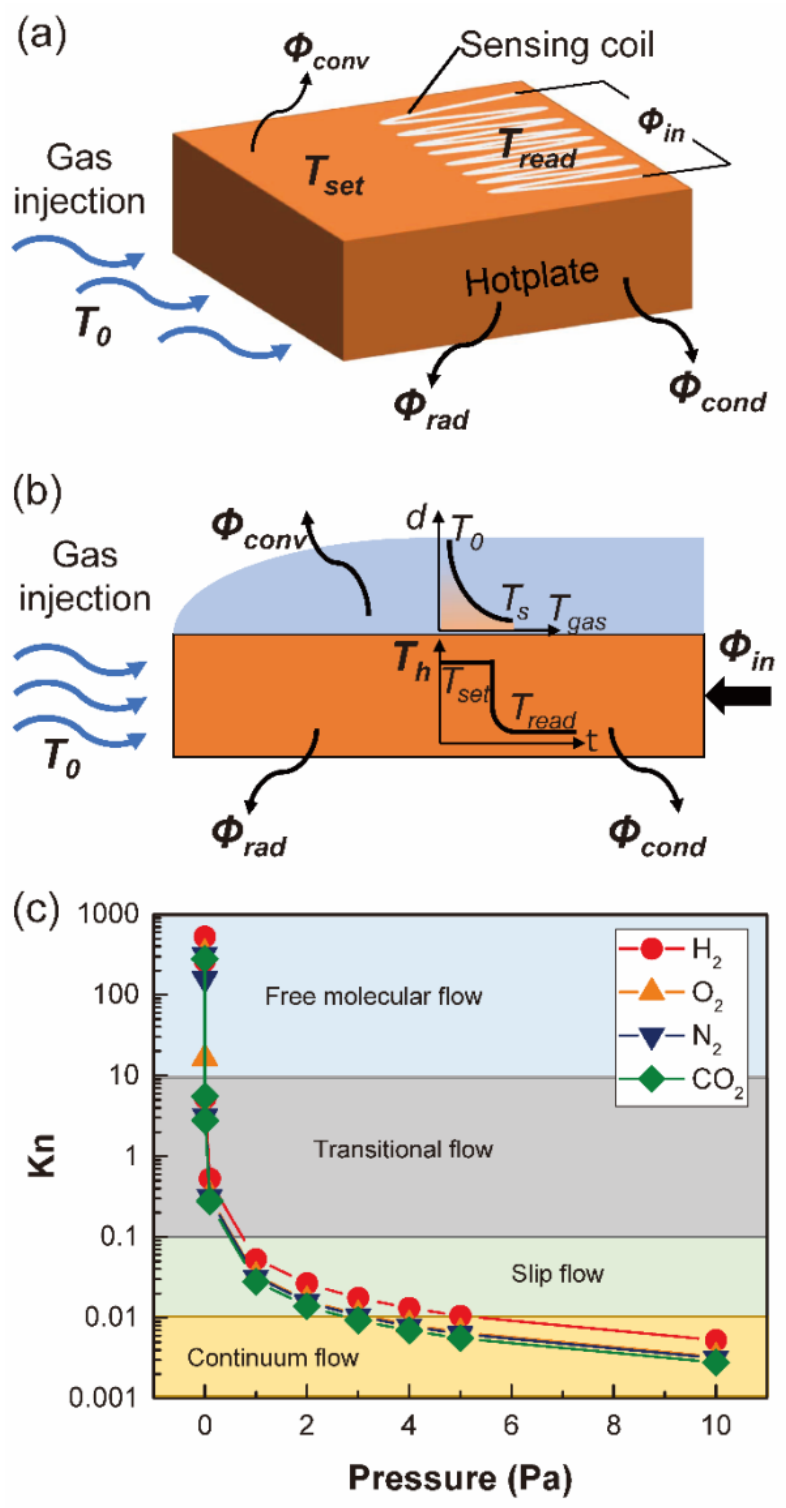

Figure 2 | Theoretical modeling of the gas effect in ETEM heating experiments. a)

Simplified model of the experiment setup. The hotplate area of the MEMS heater can be simplified as a flat plate. b) Schematic illustration of the heat transfer model for the gas-cooling effect using the heat convection on flat plate model in classic heat transfer theory. The temperature of the gas on the surface is $T_{s}$, the temperature of the hotplate is $T_{h}$. c) Plot of the Knudsen number over gas pressure for the tested gases in ETEM. 

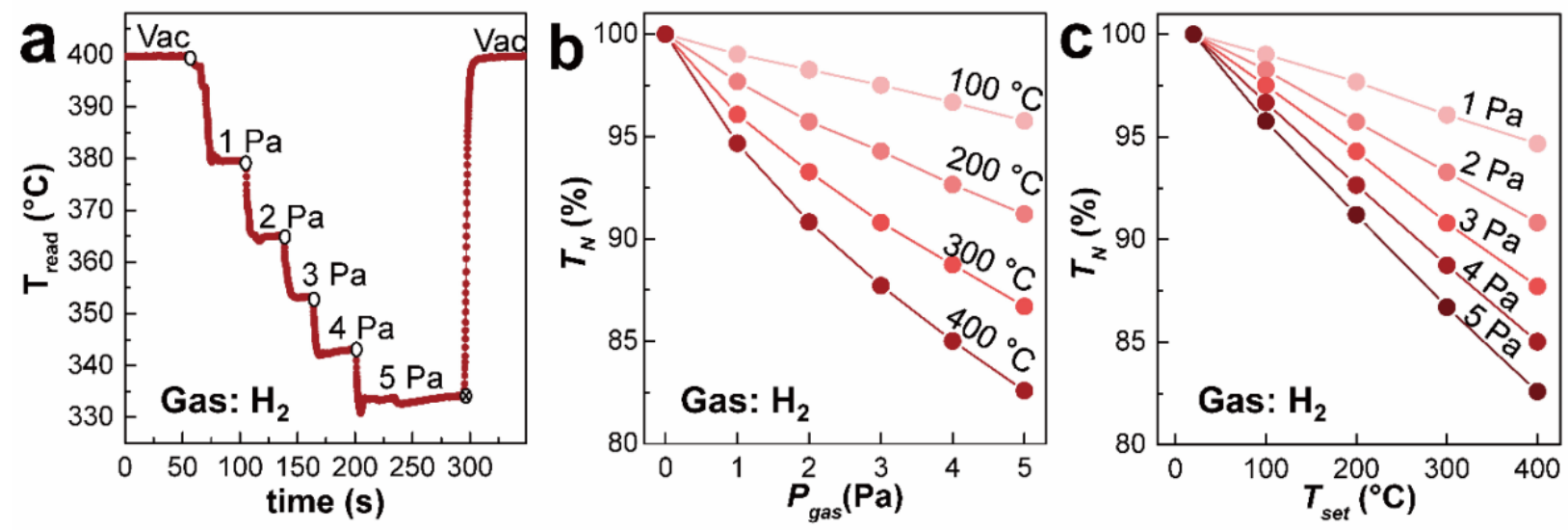

Figure 3 | Effects of gas pressure and set temperature under $\mathbf{H}_{2}$ gas flow. a) The real temperature $\left(T_{\text {read }}\right)$ decreases with increasing $\mathrm{H}_{2}$ gas pressure $P_{\text {gas. }}$ b) Normalized temperature $\left(T_{N}\right)$ decreases linearly with increasing gas pressure $P_{\text {gas }}$ under various initial temperatures $T_{\text {set. }}$ c) Normalized temperature $\left(T_{N}\right)$ decreases linearly with set temperature $T_{\text {set }}$ under different gas pressure.
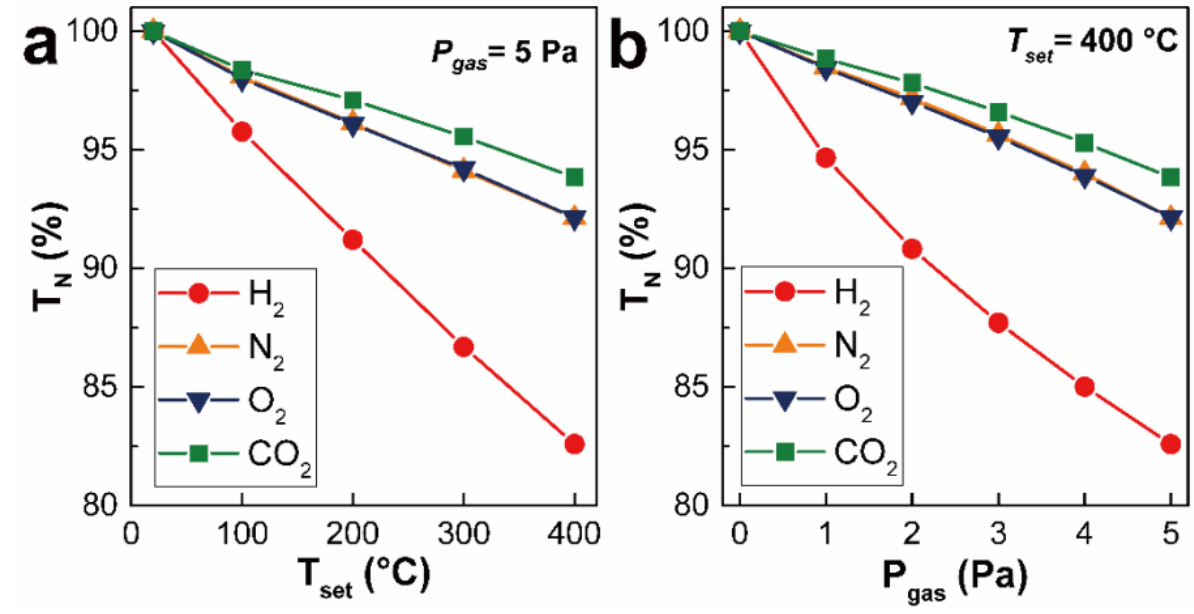

Figure $4 \mid$ Effect of gas species. a) Evolution of $T_{N} v s . P_{\text {gas }}$ under $T_{\text {set }}=400^{\circ} \mathrm{C}$ for four different gases. b) Evolution of $T_{N} v s . T_{\text {set }}$ under $P_{\text {gas }}=5 \mathrm{~Pa}$ for four different gases. 

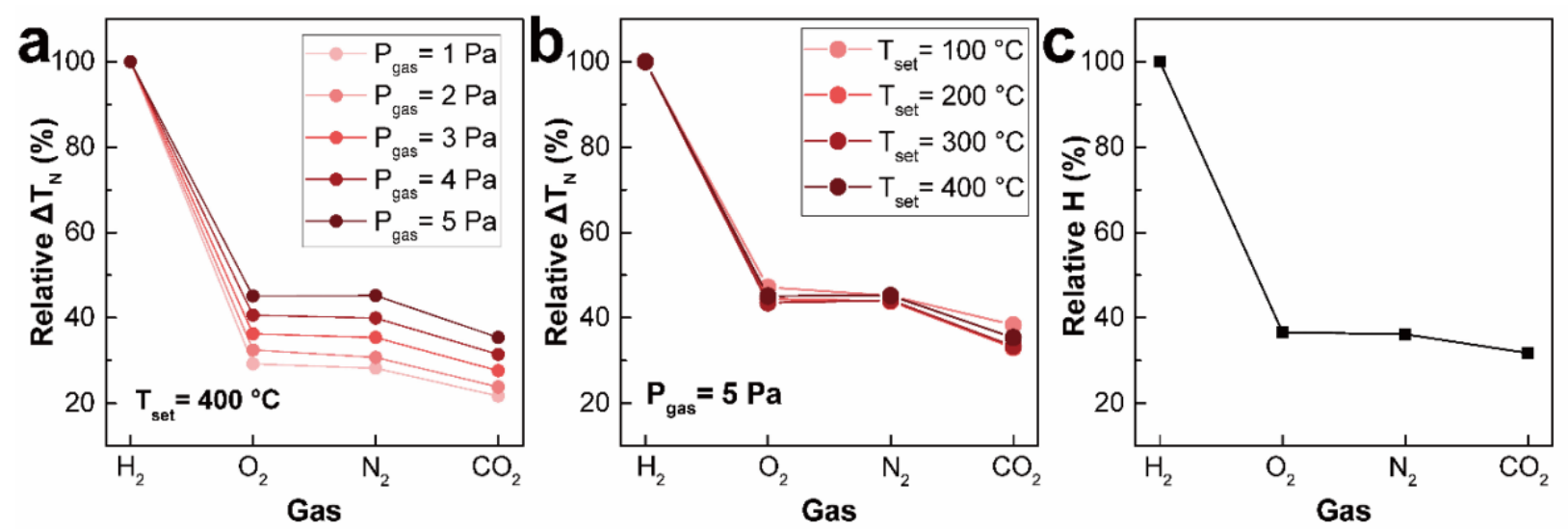

Figure 5 | The relationship between relative normalized temperature drop $\left(\Delta T_{R N}\right.$

$\left.=\Delta T_{N, \text { gas }} \Delta \boldsymbol{\Delta} \boldsymbol{T}_{N, H 2}\right)$ and the relative gas cooling ability $\left(\boldsymbol{H}_{\boldsymbol{R}}=\boldsymbol{H}_{\text {gas }} / \boldsymbol{H}_{\boldsymbol{H} 2}\right)$. a) $\Delta T_{R N} v s$. gas species with $P_{\text {gas }}=1 \sim 5 \mathrm{~Pa}$ and $T_{\text {set }}=400{ }^{\circ} \mathrm{C}$. b) $\Delta T_{R N} v s$. gas species with $T_{\text {sel }}=100 \sim 400{ }^{\circ} \mathrm{C}$ and $P_{\text {gas }}=5$ Pa. c) $H_{R} v s$. gas species under ambient temperature and pressure.

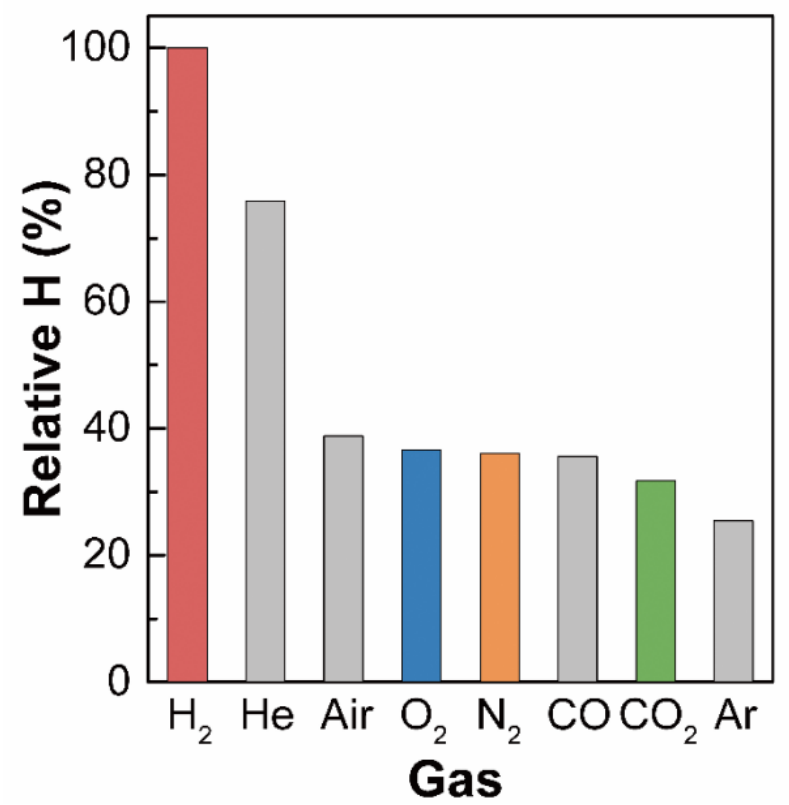

Figure 6 | Predicting the gas cooling effect of typical gas species used in ETEM by calculated relative cooling ability $\left(\boldsymbol{H}_{\boldsymbol{R}}\right)$. The predicted gas cooling effect follows the trend of $\mathrm{H}_{2}>\mathrm{He}>\mathrm{Air}>$ $\mathrm{O}_{2} \approx \mathrm{N}_{2} \approx \mathrm{CO}>\mathrm{CO}_{2}>$ Ar. 
